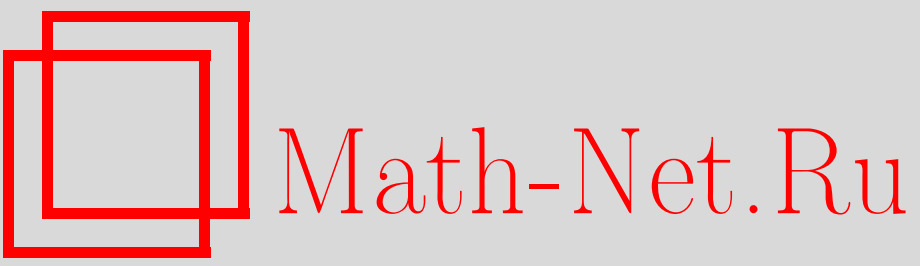

С. А. Польшин, Обобщенные когерентные состояния для массивных бесспиновых полей в пространстве де Ситтера, ТМФ, 1999, том 121, номер 2, 258-263

DOI: https://doi.org/10.4213/tmf806

Использование Общероссийского математического портала Math-Net.Ru подразумевает, что вы прочитали и согласны с пользовательским соглашением

http://www.mathnet.ru/rus/agreement

Параметры загрузки:

IP : 54.197 .217 .227

26 апреля 2023 г., 15:21:51 
ТЕОРЕТИЧЕСКАЯ

И МАТЕМАТИЧЕСКАЯ

ФИЗИКА

Том 121, № 2

ноябрь, 1999

(C) 1999 г.

С. А. Польшин ${ }^{*}$

\section{ОБОБЩЕННЫЕ КОГЕРЕНТНЫЕ СОСТОЯНИЯ ДЛЯ МАССИВНЫХ БЕССПИНОВЫХ ПОЛЕЙ В ПРОСТРАНСТВЕ ДЕ СИТТЕРА}

При помощи конформного действия группы де Ситтера $S O(4,1)$ на трехмерном евклидовом пространстве построены две различные системы обобщенных когерентных состояний для группы де Ситтера, имеющие физический смысл частиц и античастиц в пространстве де Ситтера. Скалярное произведение двух когерентных состояний является двухточечной функцией в пространстве де Ситтера.

\section{1. ВВЕДЕНИЕ}

Обобшенные когерентные состояния (KC) для произвольной группы Ли, впервые введенные в работе [1], хорошо зарекомендовали себя при решении многих физических задач. Пусть некоторое представление группы Ли $\mathcal{G}$ действует на некотором линейном векторном пространстве $H$. Рассмотрим подгруппу $\mathcal{H} \subset \mathcal{G}$ и выберем по одному представителю $g_{\xi} \in \mathcal{G}$ в каждом классе смежности $\xi \in \mathcal{G} / \mathcal{H}$. Тогда система КС будет получаться при действии преобразований в $H$, соответствующих преобразованиям $g_{\xi}$ для произвольных $\xi$, на некоторый $H$-инвариантный с точностью до фазы вектор $\left|\psi_{0}\right\rangle \in H$. Важнейшим свойством системы КС является ее инвариантность относительно некоторого представления группы $\mathcal{G}$, действуюшего в пространстве $H$. Обшие свойства систем $\mathrm{KC}$, их построение для конкретных пространств $\mathcal{G} / \mathcal{H}$ и их различные применения широко исследовались (см. книгу [2] и обзор [3]). Одним из таких применений является $\mathcal{G}$-инвариантное описание частиц в симметрических пространствах вида $\mathcal{G} / \mathcal{H}^{\prime}$, где, вообще говоря, $\mathcal{H} \subseteq \mathcal{H}^{\prime} ;$ наиболее физически важными искривленными симметрическими пространствами являются пространства де Ситтера (дС) 1-го рода $S O(4,1) / S O(3,1)$ и 2-го рода $S O(3,2) / S O(3,1)$ и их многомерные обобщения вида $S O(p+1, q) / S O(p, q)$. В [2] уже были построены $\mathrm{KC}$ для пространства $S O(n, 1) / S O(n)$. В работах $[4,5]$ рассматривались КС для пространств $S O(3,2) / S O(3) \times S O(2)$ и $S O(3,2) / S O(2) \times S O(2)$. Эти КС отвечают квантово-механическому описанию частицы в пространстве дС 2-го рода в рамках картины фазового пространства; аналогичное рассмотрение для двумерного случая проведено в [6].

${ }^{*}$ Харьковский государственный университет, Харьков, Украина. E-mail: polshin@kink.univer. kharkov.ua 
Настоящая работа посвящена построению $\mathrm{KC}$, для которых $\mathcal{G} / \mathcal{H}$ является пространством дС и которые соответствуют массивным бесспиновым клейн-гордоновским частицам в пространстве дС (так что $\mathcal{H}=\mathcal{H}^{\prime}$ ). Это отождествление проводится при помощи сравнения с “плоскими волнами” для указанного класса частиц в пространстве дС [7]. План настоящей работы следуюший. В разделе 2 рассматриваются некоторые свойства группы дС и ее необходимые в дальнейшем представления. В разделе 3 построены две системы КС, связанные с координатами Леметра и отвечающие частицам и античастицам. Показано, что двухточечные функции в пространстве дС, изучавшиеся в работах $[7,8]$, являются просто скалярным произведением двух КС.

Индексы $\mu, \nu, \ldots$ всюду пробегают значения $0, \ldots, 3 ;$ индексы $i, k, \ldots$ пробегают значения $1,2,3$. У 4-векторов индексы могут опускаться; тогда точка "." обозначает скалярное произведение при помощи галилеева метрического тензора $\eta_{\mu \nu} ; 3$-векторы в безындексных обозначениях выделяются жирным шрифтом.

\section{2. ГРУППА ДЕ СИТТЕРА И ЕЕ ПРЕДСТАВЛЕНИЯ}

Группой симметрии пространства дС является $\mathcal{G}=S O(4,1)$ - группа преобразований фиктивного пятимерного вешественного пространства с координатами $x^{0}, \ldots, x^{3}, x^{5}$, которые оставляют инвариантной квадратичную форму $\left(x^{5}\right)^{2}-\eta_{\mu \nu} x^{\mu} x^{\nu}=R^{2}$, где введен галилеев метрический тензор $\eta_{\mu \nu}=\operatorname{diag}(+1,-1,-1,-1)$ и $R$ - радиус пространства дС. Далее мы будем считать, что $x^{5}>0$; тогда координаты $x^{\mu}$ покрывают половину пространства дС. Действие произвольного элемента группы дС $g \in \mathcal{G}$ на пространстве дС мы обозначим через $x \mapsto x_{g}$. Стационарной подгруппой произвольной точки пространства дС является $\mathcal{H}=S O(3,1)$, поэтому пространство дС можно отождествить со множеством классов смежности $\mathcal{G} / \mathcal{H}$.

Обозначим через $J_{5 \mu}$ генераторы гиперболических врашений в плоскостях $(5 \mu)$ и $P_{\mu}=R^{-1} J_{5 \mu}$, тогда коммутационные соотношения имеют вид

$$
\begin{aligned}
{\left[P_{\mu}, P_{\nu}\right] } & =R^{-2} J_{\mu \nu} \\
{\left[P_{\mu}, J_{\nu \rho}\right] } & =\eta_{\mu \nu} P_{\rho}-\eta_{\mu \rho} P_{\nu} \\
{\left[J_{\mu \nu}, J_{\rho \sigma}\right] } & =\eta_{\mu \sigma} J_{\nu \rho}+\eta_{\nu \rho} J_{\mu \sigma}-\eta_{\mu \rho} J_{\nu \sigma}-\eta_{\nu \sigma} J_{\mu \rho},
\end{aligned}
$$

причем $J_{\mu \nu}$ являются генераторами подгруппы $\mathcal{H}$. Построим операторы

$$
\Pi_{i}^{ \pm}=P_{i} \pm \frac{1}{R} J_{0 i}
$$

Нетрудно показать, что

$$
\left[\Pi_{i}^{+}, \Pi_{k}^{+}\right]=\left[\Pi_{i}^{-}, \Pi_{k}^{-}\right]=0 .
$$

Операторы $\boldsymbol{\Pi}^{+}, \boldsymbol{\Pi}^{-}, P^{0}$ и $J_{i k}$ можно взять в качестве нового набора генераторов группы дС; порождаемые ими подгруппы группы дС мы обозначим через $\mathcal{T}^{+}, \mathcal{T}^{-}, \mathcal{T}^{0}$ и $\mathcal{R}=$ 
$S O(3)$. Заметим, что в силу (2) группы $\mathcal{T}^{ \pm}$абелевы. Коммутационные соотношения, помимо (2), имеют вид

$$
\begin{aligned}
{\left[\Pi_{i}^{+}, \Pi_{k}^{-}\right] } & =-\frac{2}{R} P^{0} \delta_{i k}+\frac{2}{R^{2}} J_{i k}, & {\left[P^{0}, J_{i k}\right] } & =0, \\
{\left[\Pi_{i}^{ \pm}, J_{k l}\right] } & =\Pi_{k}^{ \pm} \delta_{i l}-\Pi_{l}^{ \pm} \delta_{i k}, & {\left[P^{0}, \boldsymbol{\Pi}^{ \pm}\right] } & = \pm \frac{1}{R} \boldsymbol{\Pi}^{ \pm} .
\end{aligned}
$$

2.1. Скалярное представление. Генераторы скалярного представления суть векторы Киллинга пространства дС:

$$
P_{\mu}=\chi \frac{\partial}{\partial x^{\mu}}, \quad J^{\mu \nu}=\left(x^{\mu} \eta^{\nu \sigma}-x^{\nu} \eta^{\mu \sigma}\right) \frac{\partial}{\partial x^{\sigma}}
$$

где $\chi=\sqrt{1+(x \cdot x) / R^{2}}$. Поэтому в силу (2) генераторы (1) в скалярном представлении будут производными по некоторым новым координатам:

$$
\boldsymbol{\Pi}^{ \pm}=\frac{\partial}{\partial \mathbf{y}_{ \pm}}
$$

Таким образом, в пространстве дС можно ввести две различные системы координат Леметра. Подставляя (5) и (6) в правую и левую части равенства (1), соответственно, получаем связь $\mathbf{y}_{ \pm}$с $x^{\mu}$. Новую временную координату, не зависяшую от $\mathbf{y}_{ \pm}$, мы обозначим через $y_{ \pm}^{0}=\tau_{ \pm}$; тогда преобразования от старых координат к новым имеют вид

$$
\mathbf{y}_{ \pm}=\mathbf{x} e^{\mp \tau_{ \pm} / R}, \quad e^{ \pm \tau_{ \pm} / R}=\chi \pm \frac{x^{0}}{R}
$$

Оператор $P_{0}$ в новых координатах следующий:

$$
P_{0}=\frac{\partial}{\partial \tau_{ \pm}} \mp \frac{1}{R} \mathbf{y}_{ \pm} \frac{\partial}{\partial \mathbf{y}_{ \pm}}
$$

Конечные преобразования из групп $\mathcal{T}^{ \pm}$и $\mathcal{T}^{0}$, которые мы обозначим через $\Theta_{ \pm}$и $\Theta_{0}$, действуют в скалярном представлении следующим образом:

$$
\begin{aligned}
& g=\Theta_{ \pm}(\mathbf{a}) \equiv \exp \left(\boldsymbol{\Pi}^{ \pm} \mathbf{a} R\right):\left\{\begin{array}{l}
\mathbf{y}_{ \pm} \longmapsto \mathbf{y}_{ \pm}^{\prime}=\mathbf{y}_{ \pm}+\mathbf{a} R, \\
\tau_{ \pm} \longmapsto \tau^{\prime} \tau_{ \pm}
\end{array}\right. \\
& g=\Theta_{0}(\varepsilon) \equiv \exp \left(P_{0} \varepsilon R\right):\left\{\begin{array}{l}
\mathbf{y}^{\prime}{ }_{ \pm}=\mathbf{y}_{ \pm} e^{\mp \varepsilon} \\
\tau^{\prime}{ }_{ \pm}=\tau_{ \pm}+\varepsilon R
\end{array}\right.
\end{aligned}
$$

Мы будем считать, что преобразования действуют справа налево. 
2.2. Конформное представление. Группа дС изоморфна группе конформных преобразований вешественного трехмерного пространства, вектор которого мы обозначим через w. Существуют две различные конформные реализации группы дС, одна из которых соответствует выбору верхнего, а другая - нижнего знака в формулах, приведенных в дальнейшем. Генераторы имеют вид

$$
\begin{aligned}
R \boldsymbol{\Pi}^{\mp} & =-\frac{\partial}{\partial \mathbf{w}}, & R \boldsymbol{\Pi}^{ \pm} & =w^{2} \frac{\partial}{\partial \mathbf{w}}-2 \mathbf{w}\left(\mathbf{w} \frac{\partial}{\partial \mathbf{w}}\right), \\
R P_{0} & = \pm \mathbf{w} \frac{\partial}{\partial \mathbf{w}}, & J_{i k} & =w_{k} \frac{\partial}{\partial w_{i}}-w_{i} \frac{\partial}{\partial w_{k}} .
\end{aligned}
$$

Они удовлетворяют коммутационным соотношениям (2), (3) и (4). Конечные преобразования представляются следуюшим образом:

$$
\begin{aligned}
& g=\Theta_{\mp}(\mathbf{a}): \mathbf{w}_{g}=\mathbf{w}-\mathbf{a}, \\
& g=\Theta_{ \pm}(\mathbf{a}): \mathbf{w}_{g}=\frac{\mathbf{w}+\mathbf{a} w^{2}}{1+2 \mathbf{w} \mathbf{a}+w^{2} a^{2}}, \\
& g=\Theta_{0}(\varepsilon): \mathbf{w}_{g}=\mathbf{w} e^{ \pm \varepsilon} .
\end{aligned}
$$

Отметим, что существование конформного представления группы дС является следствием обшего утверждения [9], что конформная группа евклидова $n$-мерного пространства изоморфна $S O(n+1,1)$.

2.3. Функциональное представление. Определим два различных представления группы дС, действующей на пространстве функций, зависящих от вектора w:

$$
T_{\sigma}^{ \pm}(g) f(\mathbf{w})=\left(\alpha_{\mathbf{w}}^{ \pm}(g)\right)^{\sigma} f\left(\mathbf{w}_{g^{-1}}\right),
$$

где $\sigma \in \mathbf{C}$ и

$$
\alpha_{\mathbf{w}}^{ \pm}(g)=\left|\frac{\partial w_{g}^{i}{ }^{-1}}{\partial w^{k}}\right|^{-\frac{1}{3}}= \begin{cases}1, & g \in \mathcal{T}^{\mp}, \quad g \in \mathcal{R}, \\ e^{ \pm \varepsilon}, & g=\Theta_{0}(\varepsilon), \\ 1-2 \mathbf{a w}+a^{2} w^{2}, & g=\Theta_{ \pm}(\mathbf{a}),\end{cases}
$$

действие группы дС на вектор w определено при помоши (10). Мы обозначим эти представления через $T_{\sigma}^{ \pm}$. Легко видеть, что генераторы в этих представлениях имеют вид

$$
\begin{aligned}
R \boldsymbol{\Pi}^{\mp} & =-\frac{\partial}{\partial \mathbf{w}}, & R \boldsymbol{\Pi}^{ \pm} & =w^{2} \frac{\partial}{\partial \mathbf{w}}-2 \mathbf{w}\left(\mathbf{w} \frac{\partial}{\partial \mathbf{w}}\right)+2 \sigma \mathbf{w}, \\
R P_{0} & = \pm\left(\mathbf{w} \frac{\partial}{\partial \mathbf{w}}-\sigma\right), & J_{i k} & =w_{k} \frac{\partial}{\partial w_{i}}-w_{i} \frac{\partial}{\partial w_{k}}
\end{aligned}
$$

В пространстве представления $T_{\sigma}^{ \pm}$определим скалярное произведение следуюшим обpaзом:

$$
\left\langle f_{1} \mid f_{2}\right\rangle=\int_{\mathbf{R}^{3}} d^{3} \mathbf{w} f_{1}^{*}(\mathbf{w}) f_{2}(\mathbf{w}) .
$$


Нетрудно показать, что оно будет инвариантным в пространстве дС при

$$
\sigma=\sigma_{0} \equiv-\frac{3}{2}-i \mu R, \quad \mu \in \mathbf{R} .
$$

Поэтому представление $T_{\sigma_{0}}^{ \pm}$- унитарное; оно, однако, не является неприводимым, т.к. мы не делаем предположения о квадратичной интегрируемости функций из его носителя, и потому оно содержит инвариантное подпространство квадратично интегрируемых функций.

\section{3. КОГЕРЕНТНЫЕ СОСТОЯНИЯ}

Вложением пространства дС в группу дС называется отображение $\mathcal{G} / \mathcal{H} \ni y_{ \pm} \mapsto$ $g_{y_{ \pm}} \in \mathcal{G}$ такое, что для произвольного $g_{1} \in \mathcal{G}$ справедливо равенство

$$
g_{1} g_{y_{ \pm}}=g_{y_{ \pm}^{\prime}} h, \quad h \in \mathcal{H}, \quad y_{ \pm}^{\prime}=\left(y_{ \pm}\right)_{g_{1}} .
$$

Покажем, что соотношение

$$
g_{y_{ \pm}}=\Theta_{ \pm}\left(\frac{\mathbf{y}_{ \pm}}{R}\right) \Theta_{0}\left(\frac{\tau_{ \pm}}{R}\right)
$$

определяет вложение. Действительно, преобразование $g_{y_{ \pm}}$, задаваемое равенством (13), переводит начало координат в точку с координатами $y_{ \pm}$. Поэтому преобразования $g_{1} g_{y_{ \pm}}$и $g_{y_{ \pm}^{\prime}}$ оба переводят начало координат в точку $y_{ \pm}^{\prime}$; следовательно, преобразование $\left(g_{1} g_{y_{ \pm}}\right)^{-1} g_{y_{ \pm}^{\prime}}$ оставляет начало координат на месте и потому принадлежит $\mathcal{H}$. Вектор $\left|\psi_{0}\right\rangle$, лоренц-инвариантный относительно действия представления $T_{\sigma}^{ \pm},-$это вектор

$$
\left|\psi_{0}\right\rangle=\left(1-w^{2}\right)^{\sigma}
$$

его лоренц-инвариантность можно показать, действуя на него генераторами (11) и (12) и выражая $J_{0 i}$ через $\Pi^{+}$и $\Pi^{-}$при помоши (1). Теперь можно построить систему КС для представлений $T_{\sigma}^{ \pm}$:

$$
|x, \pm ; \sigma\rangle=T_{\sigma}^{ \pm}\left(g_{y_{ \pm}(x)}\right)\left|\psi_{0}\right\rangle,
$$

где координаты Леметра считаются зависящими от координат $x^{\mu}$ при помощи преобразований (7). Явным выражением для $\mathrm{KC}$ как функций от $\mathbf{w}$ будет

$$
|x, \pm ; \sigma\rangle \equiv \Phi_{\mathbf{w}}^{ \pm}(x ; \sigma)=\left(1-w^{2}\right)^{\sigma} \varphi_{k_{\mathbf{w}}}^{ \pm}(x ; \sigma),
$$

где

$$
\begin{aligned}
& \varphi_{k}^{ \pm}(x ; \sigma)=\left(\chi \pm \frac{k \cdot x}{R}\right)^{\sigma}, \\
& k_{\mathbf{w}}^{\mu}=\left(\frac{1+w^{2}}{1-w^{2}}, \pm \frac{2 \mathbf{w}}{1-w^{2}}\right),
\end{aligned}
$$


так что $k_{\mathbf{w}} \cdot k_{\mathbf{w}}=1$. Функции $\varphi_{k}^{ \pm}\left(x ; \sigma_{0}\right)$ при $k^{0}>0$ и $k \cdot k=1$ являются "плоскими волнами" для частиц (знак + ) и античастищ (знак - ) массы $\mu$ [7]. Поэтому системы $\mathrm{KC} \mathrm{(14)} \mathrm{при} \sigma=\sigma_{0}$ и $w^{2}<1$ соответствуют частицам (знак + ) и античастицам (знак -); если же $w^{2}>1$, то это соответствие будет обратным.

Вычисляя якобиан перехода от $\mathbf{w}$ к $\mathbf{k}_{\mathbf{w}}$, получаем выражение для скалярного произведения двух $\mathrm{KC} \mathrm{в} \mathrm{представлении} T_{\sigma_{0}}^{ \pm}$:

$$
\begin{aligned}
\left\langle\stackrel{2}{x}, \pm ; \sigma_{0} \mid \frac{1}{x}, \pm ; \sigma_{0}\right\rangle & \equiv \int_{\mathbf{R}^{3}} d^{3} \mathbf{w} \Phi_{\mathbf{w}}^{ \pm}\left(\frac{1}{x} ; \sigma_{0}\right) \Phi_{\mathbf{w}}^{ \pm}\left(\stackrel{2}{x} ; \sigma_{0}^{*}\right)= \\
& = \pm \frac{1}{8} \int_{k^{0}>0} \frac{d^{3} \mathbf{k}}{k^{0}}\left(\varphi_{k}^{+}\left(\stackrel{1}{x} ; \sigma_{0}\right) \varphi_{k}^{+}\left(\stackrel{2}{x} ; \sigma_{0}^{*}\right)-\varphi_{k}^{-}\left(\stackrel{1}{x} ; \sigma_{0}\right) \varphi_{k}^{-}\left(\stackrel{2}{x} ; \sigma_{0}^{*}\right)\right)
\end{aligned}
$$

Действительно, область $\mathbf{R}^{3} \backslash S_{w^{2}=1}^{2}$ при отображении $\mathbf{w} \mapsto k_{\mathbf{w}}$ непрерывно отображается на обе полы гиперболоида $k \cdot k=1$. При $w^{2}=1$ подынтегральное выражение в первом интеграле в (15) не имеет особенностей при $\stackrel{1}{x} \cdot \stackrel{1}{x}>0$ и $\stackrel{2}{x} \cdot \stackrel{2}{x}>0$. Второй интеграл в (15) совпадает с двухточечными функциями в пространстве дС, рассматривавшимися в работах $[7,8]$. Интегралы в $(15)$, вообше говоря, расходятся; это связано с тем, что функция $\left|\psi_{0}\right\rangle$ не является квадратично интегрируемой на $\mathbf{R}^{3}$. Этим интегралам можно придать смысл путем перехода в комплексное пространство дС [7] или же к обобщенным функциям [8].

Заметим, что функции $\varphi_{k}^{-}\left(x ; \sigma_{0}\right)$ при $k^{0}>0$ являются КС для пространства дС, построенными по аналогии с $\mathrm{KC}$ для пространства $S O(n, 1) / S O(n)$ [2]. Такой подход, однако, не дает возможности равноправно описывать частицы и античастицы.

Благодарности. Я благодарен Ю. П. Степановскому за ценные обсуждения, а также рецензенту за замечания, способствовавшие улучшению содержания статьи.

\section{Список литературы}

[1] A. M. Perelomov. Commun. Math. Phys. 1972. V. 26. P. 222.

[2] А. М. Переломов. Обобщенные когерентные состояния и их применения. М.: Наука, 1987.

[3] S. Twareque Ali, J.-P. Antoine, J.-P. Gazeau, U. A. Mueller. Rev. Math. Phys. 1995. V. 7. P. 1013.

[4] J.P. Gazeau. Coherent States for Desitterian and Einsteinian Relativities. In: Selected Topics in Quantum Field Theory and Mathematical Physics. Eds. J. Niederle, J. Fischer. Singapore: World Sci., 1990. P. 182.

[5] A. El Gradechi, S. De Bièvre. Ann. Phys. (USA). 1994. V. 235. P. 1; hep-th/9210133.

[6] S. De Bièvre, A. El Gradechi. Ann. Inst. H. Poincaré. Ser. Phys. Theór. 1992. V. 57. P. 403.

[7] J. Bros, U. Moschella. Rev. Math. Phys. 1996. V. 8. P. 327; gr-qc/9511019.

[8] М. Б. Менский. Метод индуцированных представлений. Пространство-время и концепция частиц. М.: Наука, 1976.

[9] Б. А. Розенфельд. Многомерные пространства. М.: Наука, 1966.

Поступила в редакцию 2.XI.1998 г., после доработки 20.IV.1999 г. 